\title{
The size of anterior teeth in patients with gaps in the upper dental arch
}

\author{
A. Sękowska1 ${ }^{1}$ I. Dunin-Wilczyńska', R. Chałas² \\ ${ }^{1}$ Department of Jaw Orthopaedics, Medical University of Lublin, Poland \\ ${ }^{2}$ Department of Conservative Dentistry and Endodontics, Medical University of Lublin, Poland
}

[Received 2 November 2014; Accepted 18 January 2015]

\begin{abstract}
Background: The aim of this study was to assess the size of upper incisors and canines in patients with gaps in the upper dental arch, especially medium gap between upper central incisors.

Materials and methods: Diagnostic orthodontic models of 30 adult patients with full permanent dentition with diastema in the upper arch were studied. Patients with severe malocclusion, missing teeth and periodontal disease were excluded. Width-to-length (W/L) ratio of the clinical crown of the central, lateral incisors and canines for both sides was measured. Together 180 teeth were tested. The results were compared with the values indicated by Sterrett et al.

Results: In all patients, the clinical crowns of central incisors were symmetrical. In most cases, a higher W/L ratio was found, which indicates that the clinical crowns of medial incisors were too broad in relation to the length. Lateral incisors: In most cases, the ratio was the same for the right and the left side; however, a few patients had asymmetry of lateral incisors. Most of the lateral incisors had higher W/L ratios, which means that the teeth were wider than they were long; some had reduced ratios and only in one case the ratio was proper. Canines were also asymmetrical, and none of the canine exhibited perfect proportions. The vast majority showed increased W/L ratio of the clinical crown. In several cases, the W/L ratio was decreased.

Conclusions: Patients with gaps between the teeth have abnormal W/L ratio of the clinical crowns of the upper front teeth. The values were increased in the majority of cases, which indicates that the front teeth were wider than they were long in patients with gaps. Moreover, despite the disturbed W/L proportions, central incisors remained symmetrical. In contrast, lateral incisors and canines more often exhibited asymmetries. (Folia Morphol 2015; 74, 4: 493-496)
\end{abstract}

Key words: teeth size, width/length ratio, diastema

\section{INTRODUCTION}

Spaces between teeth are physiological developmental phase in deciduous and mixed dentition. In children the developmental stage with spaces between incisors after central permanent incisors eruption is called "ugly duckling" stage [3]. The most popular space between upper central incisors is called diastema, but spaces between other teeth are called tremas. Based on the position of crowns of upper central incisors, there are three different shapes of

Address for correspondence: R. Chałas, MD, PhD, Department of Conservative Dentistry and Endodontics, Medical University of Lublin, ul. Karmelicka 7, 20-081 Lublin, Poland, tel: +48 81528 7920, e-mail: renata.chalas@umlub.pl 
diastema: convergent, divergent and parallel diastema. The parallel diastema is when crowns of upper central incisors are parallel. In case of the convergent diastema, crowns of incisors are convergent but roots are divergent. In the divergent diastema it is inversely - crowns are divergent, but roots are convergent [12]. In permanent dentition after canines' eruption all gaps should be closed. According to Andrews' keys to normal occlusion, the contact points between teeth should be maintained in permanent dentition [1]. The look of teeth and smile has an impact on daily living of adolescents [14]. The aesthetic perception of a smile with gaps depends on sizes and localisation of spaces. Mild spaces between maxillary central incisors of less than $1 \mathrm{~mm}$ are not rated unattractive, but medium gaps of more than $2 \mathrm{~mm}$ are found unattractive for dentists, orthodontists and lay people [13]. The position of gaps plays a role as well. The most positive perception of anterior diastemas is when the gaps are symmetrical between lateral incisors and canines and in the situation where the diastema is localised asymmetrically between lateral incisor and canine or between central and lateral incisor. Less attractive is slight midline diastema and 2 larger diastemas between central and lateral incisors. Midline diastema is rated the least attractive [15]. Spacing in permanent dentition is almost half as common as crowding. According to Thilander et al. [22], the prevalence of gaps in permanent dentition is $23 \%$, where $3.7 \%$ are medium-size diastemas of $2 \mathrm{~mm}$ or bigger. The prevalence of midline diastemas in black people is higher and accounts for $37 \%$ [17]. There are different reasons of gaps in dental arches, for example aberrant labial frenum attachments $[6,10]$, parafunctional habits, tooth loss, periodontal disease [10], deep bites [16] or supernumerary teeth [5]. When the reason of diastema is large frenum, it is called diastema vera. In cases when diastema occurs because of too small or lacking lateral incisors or because of mesiodens - supernumerary tooth in the midline between upper central incisors - this type of diastema is called pseudodiastema [12]. But there are also gaps in dental arches without any environmental reasons. Some authors prove familial correlations and heritability of diastemas [7], but some indicate tooth size discrepancies $[10,16]$.

The purpose of this study was to analyse the width/length (W/L) ratio of upper incisors and canines in patients with gaps in the dental arch, especially the medium gap between upper central incisors.

\section{MATERIALS AND METHODS}

This study was approved by Bioethics Committee of the Medical University of Lublin (No. KE-0254/ 129/2014). Diagnostic (before treatment) orthodontic plaster models of 30 patients with maxillary central diastema were studied. Only patients with permanent dentition (DS4M2) according to Björk, that means Dental Stage 4 - fully erupted canines and premolars and $\mathrm{M} 2$ - fully erupted second molars, were studied [2]. All patients were Caucasian race. Digital calliper with accuracy of $0.01 \mathrm{~mm}$ was used for measurements. Patients with severe malocclusion, missing anterior teeth, traumatic injury, gingival recessions and periodontal disease were excluded. $\mathrm{W} / \mathrm{L}$ ratio of the clinical crowns of central incisors $(\mathrm{Cl})$, lateral incisors (LI) and canines (CA) for both sides were measured. The measurement of width was the greatest width of crown of each tooth and the measurement of length was the longest portion of the front teeth. Altogether 180 teeth were tested (60 teeth for each tooth group). The results were compared with the values indicated by Sterrett et al. [21] who studied W/L ratio of maxillary front teeth. They made alginate impressions of maxillary arch and poured in yellow dental stone. On plaster models they measured the widest mesiodistal and the longest apicocoronal portion of test teeth using callipers (with $0.05 \mathrm{~mm}$ accuracy) [21]. The mean W/L ratios published by Sterrett et al. [21] were the following: $\mathrm{Cl}-0.85$ (range $0.65-1.02$ ) in males and 0.86 (range 0.72-1.04) in females, LI - 0.76 (0.63-1.04) in males and $0.79(0.64-1.0)$ in females, CA -0.77 $(0.66-0.97)$ in males and $0.81(0.68-0.97)$ in females.

\section{RESULTS}

In the presented study, the mean W/L ratios in males vs. females were: $\mathrm{Cl}-1.0$ vs. $0.92, \mathrm{LI}-0.90$ vs. 0.83, CA -0.89 vs. 0.91 . Central incisors: in 46 incisors $\mathrm{W} / \mathrm{L}$ ratio was higher than presented by Sterrett et al. [21]. For both sides the number of teeth with higher ratio and lower ratio was the same (higher $\mathrm{W} / \mathrm{L}$ ratio $=23$, lower $\mathrm{W} / \mathrm{L}$ ratio $=7$ for each side) (Figs. 1A, B). Lateral incisors: there were differences for the right and the left side. Right lateral incisors had higher W/L ratio in 20 cases, and lower ratio in 10 cases. Left lateral incisors had higher $\mathrm{W} / \mathrm{L}$ ratio in 20 cases, proper ratio in 1 case, and lower ratio in 9 cases (Figs 2A, B). Canines: right canines had in 27 cases higher $W / L$ ratio, left canines - in 23 cases. Lower W/L ratio was in 3 cases for the right and in 7 for the left side (Figs. 3A, B). 

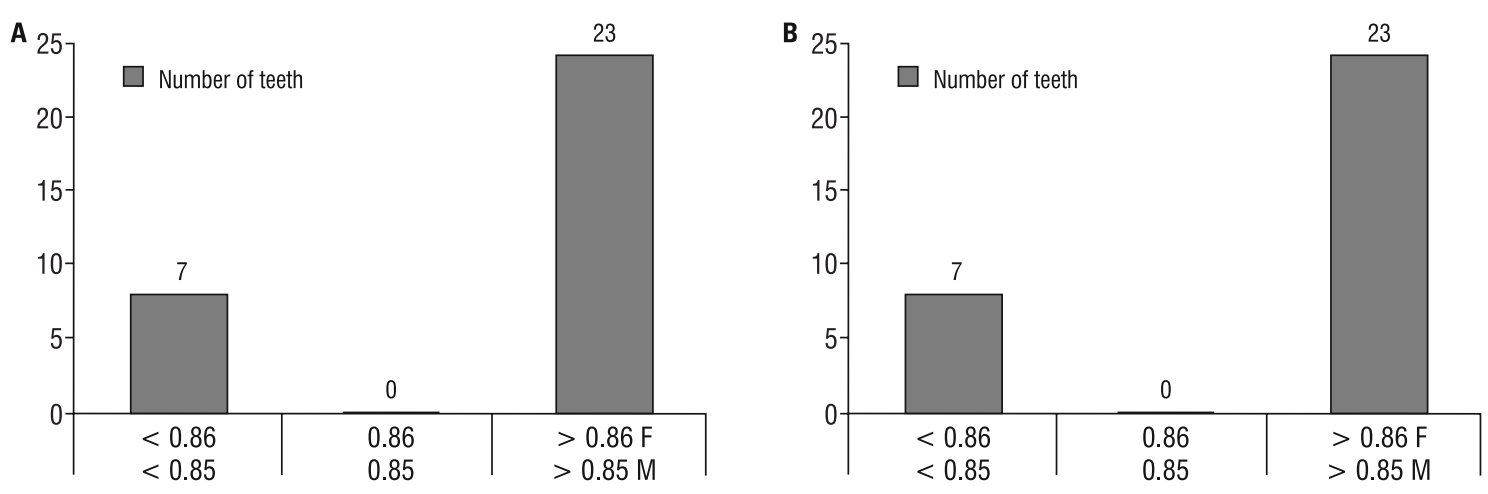

Figure 1. Width to length ratios for central incisors in examined group of patients with gaps; A. Right; B. Left; F — female; M — male.
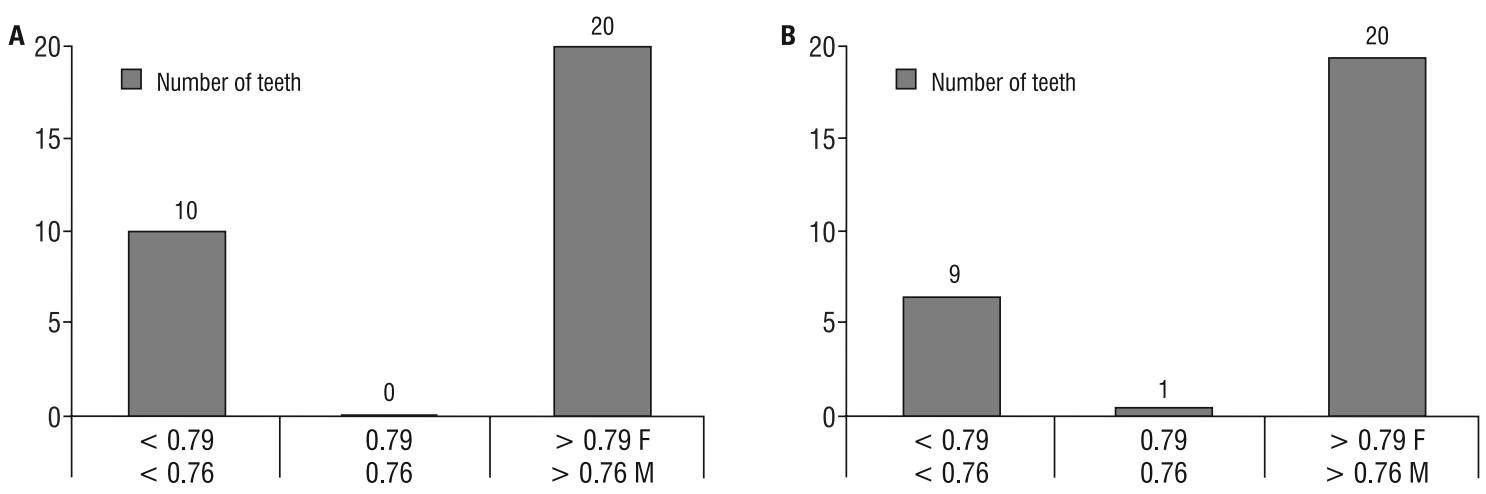

Figure 2. Width to length ratios for lateral incisors in examined group of patients with gaps; A. Right; B. Left; $F-$ female; $M-$ male.
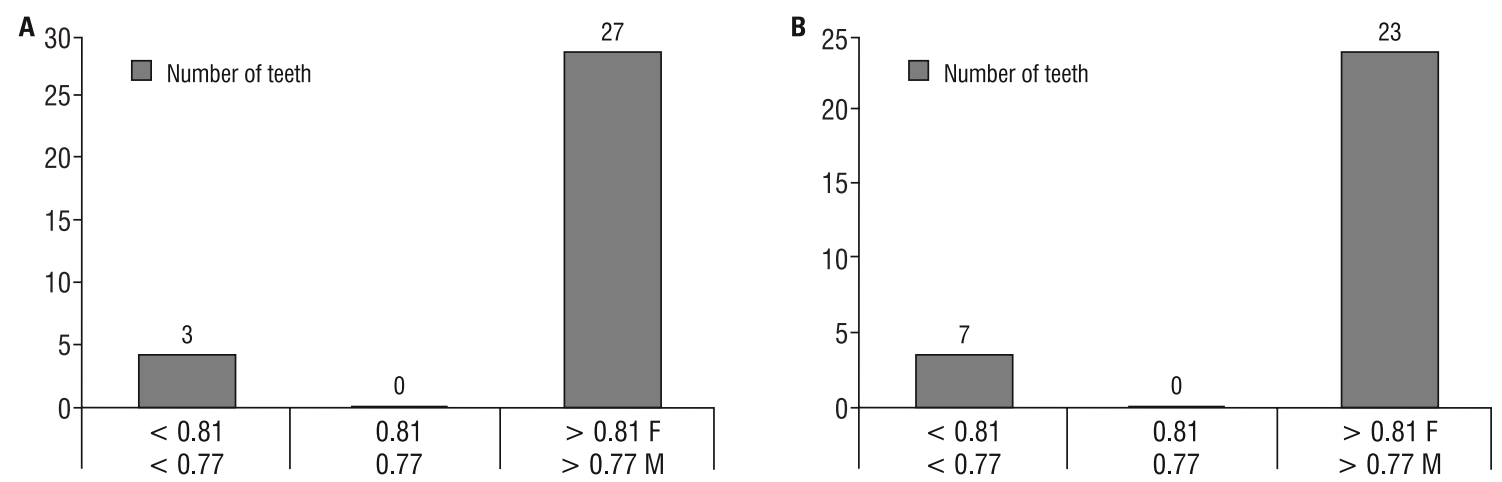

Figure 3. Width to length ratios for canines in examined group of patients with gaps; A. Right; B. Left; F — female; M — male.

\section{DISCUSSION}

The search for perfect proportions has engaged people for a long time. Even the ancient Greeks tried to find the ideal proportion which rules the world. The look of a smile is affected by two ratios: the first is the proportion of the width of upper front teeth in relation to each other and the second ratio is the height-to-width proportion [18]. The proportions of the teeth in relation to each other in a smile are described by the golden proportion otherwise called the divine proportion [11]. Because of the curvature of the dental arch, the visible widths of upper front teeth are different then real widths. According to the theory of the aesthetics of the smile, the best considered width of the lateral incisors should be $62 \%$ of the width of the central incisor and the canine apparent width $-62 \%$ of the width of the lateral incisor [20]. Ideal central incisor width-to-height proportion 
should be $75-78 \%[20]$ or $80 \%[18,20]$ and it depends on teeth length [18]. In this study the scope of the $\mathrm{W} / \mathrm{L}$ ratios was higher and amounted to $92-100 \%$.

The obtained results suggest that the examined patients with spaces in dental arches in most incisors and canines had higher $\mathrm{W} / \mathrm{L}$ ratios. Higher ratios mean that the width of crowns of teeth is bigger or the length is smaller in these patients. In most cases of $\mathrm{Cl}$, higher W/L ratio means that the clinical crowns of medial incisors were too wide in relation to the length. In several cases, the $\mathrm{W} / \mathrm{L}$ ratio was lower. Central incisors showed symmetry in results for both sides. In most cases of $\mathrm{LI}$, the ratio was the same for the right and the left side; however, a few patients had asymmetry of LI. Most of the $\mathrm{LI}$ had higher W/L ratio. This means that the teeth were wider than they were long. Some had a reduced rate. In individual cases, the ratio was normal. Canines were also asymmetrical and none of the canines exhibited perfect proportions. The vast majority showed increased $\mathrm{W} / \mathrm{L}$ ratio of the clinical crown. In several cases, the $\mathrm{W} / \mathrm{L}$ ratio was lower. Summing up, the examined group of patients with spacing in dental arches had increased transverse dimension or decreased vertical dimension in comparison with the results presented by Sterrett et al. [21]. In comparison with the research of Hasanreisoglu et al. [9], there were differences in $\mathrm{W} / \mathrm{L}$ ratios between males and females. Males had lower $W / L$ ratios than in the presented study and females had lower W/L ratio only for canines, but for incisors, the results were similar to the presented study.

In order to compare our results with Gillen et al. [8] who calculated the reverse L/W ratio, we also calculated this reverse ratios and the results were the following: mean L/W ratio for centrals was 1.089 , for laterals 1.210 , for canines 1.134 . That was similar to ratios calculated by Gillen et al. [8]: centrals $1.109 \pm$ \pm 0.104 , laterals $1.92 \pm 0.149$, canines $1.212 \pm 0.119$. Other authors $[4,19]$ who measured and compared tooth size in normal, crowded and spaced dentitions showed that there was a trend for smaller tooth widths in spaced dentitions compared with normal ones.

\section{CONCLUSIONS}

1. Canines and incisors in patients with gaps between teeth had higher W/L ratio, which means that the teeth were wider than they were long.

2. The measurement of the front teeth size can be important for dentist for planning the treatment of their patients.

\section{REFERENCES}

1. Andrews LF (1972) The six keys to normal occlusion. Am Orthod, 62: 296-309.

2. Björk A, Krebs A, Solow B (1964) A method for epidemiological registration of malocclusion. Acta Odontol Scand, 22: 27-41.

3. Broadbent BH (1937) The face of the normal child. Angle Orthod, 7: 183-208.

4. Bugaighis I, Elorfi S (2013) An odontomertric study of tooth size in normal, crowded and spaced dentitions. J Orthod Sci, 2: 95-100.

5. Campbell A, Kindelan J (2006) Maxillary midline diastema: a case report involving a combined orthodontic/maxillofacial approach. J Orthod, 33: 22-27.

6. Delli K, Livas C, Sculean A, Katsaros C, Bornstein MM (2013) Facts and myths regarding the maxillary midline frenum and its treatment: a systematic review of the literature. Quintessence Int, 44: 177-187.

7. Gass JR, Valiathan M, Tiwari HK, Hans MG, Elston RC (2003) Familial correlations and heritability of maxillary midline diastema. Am J Orthod Dentofacial Orthop, 123: 35-39.

8. Gillen RJ, Schwartz RS, Hilton TJ, Evans DB (1994) An analysis of selected normative tooth proportions. Int J Prosthodont, 7: 410-417.

9. Hasanreisoglu U, Berksun S, Aras K, Arslan I (2005) An analysis of maxillary anterior teeth: Facial and dental proportions. J Prosthet Dent, 94: 530-538.

10. Huang WJ, Creath CJ (1995) The midline diastema: a review of its etiology and treatment. Pediatr Dent, 17: 171-179.

11. Huntley HE (1970) The Divine Proportion. Dover Publications.

12. Karłowska I (2009) Zarys współczesnej ortodoncji. Wydawnictwo lekarskie PZWL, Warszawa.

13. Kokich VO, Kokich VG, Kiyak HA (2006) Perceptions of dental professionals and laypersons to altered dental esthetics: Asymmetric and symmetric situations. Am J Orthod Dentofacial Orthop, 130: 141-151.

14. Marques LS, Filogonio CA, Filogonio CB, Pereira $\sqcup$, Pordeus IA, Paiva SM, Ramos-Jorge ML (2009) Aesthetic impact of malocclusion in the daily living of Brazilian adolescents. J Orthod, 36: 152-159.

15. Noureddine A, Chabouis HF, Parenton S, Lasserre JF (2014) Laypersons' esthetic perception of various computer-generated diastemas: A pilot study. J Prosthet Dent, 112: 914-920.

16. Oesterle $\sqcup$, Shellhart WC (1999) Maxillary midline diastemas: a look at the causes. JADA, 130: 85-94.

17. Onyeaso CO. Prevalence of malocclusion among adolescents in Ibadan, Nigeria (2004) Am J Orthod Dentofacial Orthop, 126: 604-607.

18. Proffit WR, Fields HW, Sarver DM (2006) Contemporary orthodontics. Elsevier Health Sciences.

19. Puri N, Pradhan KL, Chandna A, Sehgal V, Gupta R (2007) Biometric study of tooth size in normal, crowded and spaced permanent dentitions. Am J Orthod Dentofacial Orthop, 132: 279.e7-279.e14.

20. Rosenstiel SF, Ward DH, Rashid RG (2000) Dentists' preferences of anterior tooth proportion: a web-based study. J Prosthodont, 9: 123-136.

21. Sterrett JD, Oliver T, Robinson F, Fortson W, Knaak B, Russell CM (1999) Width/length ratios of normal clinical crowns of maxillary anterior dentition in man. J Clin Periodontol, 26: 153-157.

22. Thilander B, Pena L, Infante C, Parada SS, de Mayorga C (2001) Prevalence of malocclusion and orthodontic treatment need in children and adolescents in Bogota, Columbia. An epidemiological study related to different stages of dental development. Eur J Orthodont, 23:153-167. 